\title{
LA TEORÍA DEL CAMBIO EN EL PAISAJE A PARTIR DEL CAMBIO DEL USO DE LA TIERRA Y LA COBERTURA DEL SUELO (ENFOQUE LUCC). SU UTILIDAD PARA LA HISTORIA AMBIENTAL
}

\section{THE THEORY OF CHANGE IN THE LANDSCAPE FROM LAND USE AND LAND COVER CHANGE (LUCC APPROACH). ITS USEFULNESS IN ENVIRONMENTAL HISTORY}

\author{
Andrea Montero Mora ${ }^{1}$ \\ andrea.monteromora@ucr.ac.cr \\ Ronny Viales Hurtado ${ }^{2}$ \\ ronny.viales@ucr.ac.cr
}

Fecha de recepción: 30 julio 2014 - Fecha de aceptación: 23 julio 2015

\begin{abstract}
Resumen
La teoría del cambio del paisaje desde el enfoque "Land-Use and Land-Cover-Change" (LUCC) explica la relación que existe entre las actividades humanas y la evolución del paisaje, a partir de dos procesos: la agriculturización y la urbanización. Propone estudiar dos dinámicas que se generan a partir de la interacción entre los procesos humanos y biofísicos; a saber: el cambio en el uso de la tierra y el cambio en la cobertura del suelo y su relación con el cambio ambiental global. La cobertura del suelo se define como el estado biofísico de la superficie de la tierra o las características del terreno; y el uso de la tierra, como la manera en que la sociedad utiliza el suelo para desarrollar actividades económicas. El cambio en la cobertura del suelo se define como la transformación de la apariencia física de un paisaje determinado y el cambio en el uso de la tierra, como los cambios en el manejo de la tierra y sus atributos. El presente artículo tiene como propósito introducir los elementos básicos del enfoque para reconocer sus alcances y limitaciones desde la perspectiva de la historia ambiental.

Palabras claves: paisaje, agricultura, agriculturización, historia ambiental, enfoque LUCC.
\end{abstract}

\begin{abstract}
The theory of landscape change from the "Land-Use and Land-Cover-Change" (LUCC) approach explains the relationship between human activities and landscape evolution from two processes: agriculture and urbanization. Submit study two dynamics generated from the interaction between human and biophysical processes namely the change in land use and change in land cover and its relationship to global environmental change. The ground cover is defined as the biophysical state of the surface of land or land features, and land use, as the way in which society uses the ground for the development of economic activities. The change in land cover is defined as the transformation of the physical
\end{abstract}

1 Profesora de la Escuela de Historia e Investigadora en el Centro de investigaciones Históricas de América Central (CIHAC), de la Universidad de Costa Rica.

2 Docente de la Escuela de Historia, del Posgrado Centroamericano en Historia e Investigador en el Centro de Investigaciones Históricas de América Central (CIHAC), de la Universidad de Costa Rica. 
appearance of a particular landscape, and the change in land use, such as changes in land management and its attributes. This article aims to introduce the basic elements of the approach to recognize their scope and limitations from the perspective of environmental history.

Key Words: landscape, agriculture, agricultural development, environmental history, LUCC approach.

\section{Introducción}

El enfoque LUCC: una propuesta para estudiar el paisaje que puede adoptarse para construir una visión retrospectiva

El enfoque "Land-Use and Land-Cover-Change" (LUCC) es una propuesta reciente. Nació en la década de 1990, bajo la iniciativa de dos programas: el Programa Internacional Geosfera Biosfera (IGBP) (Recuperado de: http://www.igbp-es.org/) y el Programa de Dimensiones Humanas (HDP). Ambos programas se interesaron en estudiar el cambio en la cobertura del suelo y el uso de la tierra, con el propósito de mejorar el conocimiento científico sobre la evolución del paisaje y su relación con el cambio global. El enfoque propone un análisis que solo se puede lograr desde la multi-disciplinariedad y la trans-disciplinariedad, pues se requiere integrar variables biofísicas, políticas y socioeconómicas para explicar las transformaciones en el paisaje (Turner, Skole, Sanderson, Günther, Fresco \& Leemans, 1995). Desde nuestro punto de vista, es fundamental complementar esas variables con elementos histórico-ambientales, con sus trayectorias y sus contextos cambiantes.

Cuadro 1

Focos de investigación del enfoque LUCC

\begin{tabular}{|c|}
\hline DINÁMICA DEL \\
USO DE LA TIERRA \\
\hline • Se pretende entender el \\
proceso de cambio en el uso \\
de la tierra. \\
- Se pretende conocer las \\
decisiones con respecto al \\
uso de la tierra. \\
\end{tabular}

\begin{tabular}{|c|}
\hline DINÁMICA DE LA \\
COBERTURA DEL \\
SUELO \\
\hline - Se pretende entender el \\
proceso de cambio en la \\
cobertura del suelo. \\
- Se pretende explicar la tasa \\
de cambio de la cobertura, la \\
ubicación y los atributos \\
espaciales y ambientales que \\
explican el cambio. \\
\hline
\end{tabular}

\begin{tabular}{|c|}
\hline MODELACIÓN \\
REGIONAL Y \\
GLOBAL \\
\hline $\begin{array}{c}\text { - Se pretende analizar y } \\
\text { predecir los cambios en el } \\
\text { uso de la tierra y la } \\
\text { cobertura del suelo. } \\
\text { - Se pretende hacer énfasis en } \\
\text { las fuerzas impulsoras de } \\
\text { cambio. }\end{array}$ \\
\hline
\end{tabular}

Fuente: Elaboración propia a partir de (Turner, Skole, Sanderson, Günther, Fresco \& Leemans, 1995).

Desde sus inicios, el enfoque LUCC definió tres focos de investigación que se resumen en el Cuadro No. 1. Para una mejor comprensión de los focos propuestos, resulta pertinente aclarar algunas definiciones. De acuerdo con la propuesta LUCC, la cobertura del suelo es el estado biofísico de la superficie de la tierra o las características del terreno. El uso de la tierra es la forma en que la sociedad, a través de actividades económicas, utiliza la cobertura. El cambio en el uso de la tierra se refiere a los cambios en el manejo de esta y sus atributos. Finalmente, el cambio en la cobertura del suelo se 
refiere a la transformación de la apariencia física de un paisaje determinado (Guhl, 2008). La cobertura del suelo es entonces lo que se encuentra sobre el terreno en un momento dado y el uso de la tierra se refiere a los propósitos y la manera en que se utiliza el terreno (Turner, Skole, Sanderson, Günther, Fresco \& Leemans, 1995).

\section{El enfoque LUCC y su categorización del paisaje}

Estudiar el cambio en la cobertura del suelo y el uso de la tierra y su relación con los paisajes no es sencillo. Por ello es necesario comprender ¿qué entiende el enfoque LUCC por paisajes? y ¿cómo analizan su transformación? En la propuesta, el paisaje ha sido agrupado en tres grandes categorías analíticas: los paisajes fabricados, los paisajes domesticados y los paisajes naturales. A continuación, presentamos una breve caracterización de cada tipo de paisaje:

1. Los paisajes fabricados: son aquellos lugares valorados como desarrollados y en los cuales los cambios han sido provocados por variables asociadas a las actividades humanas, que requieren a su vez un uso intensivo de energía, principalmente, a partir de combustibles fósiles.

2. Los paisajes domesticados: son aquellos en los que el Sol es la fuente básica de energía y el uso de energía es también intensivo. Las actividades humanas tienen influencia directa sobre los cambios que ocurren en esos lugares, pero en menor grado que los paisajes fabricados.

3. Los paisajes naturales: son ambientes autosostenibles controlados por procesos biofísicos, pero con influencia antropogénica, donde la estructura del ecosistema continúa controlada por procesos de carácter natural (Guhl, 2008).

Figura 1

Las cinco interrogantes del enfoque LUCC

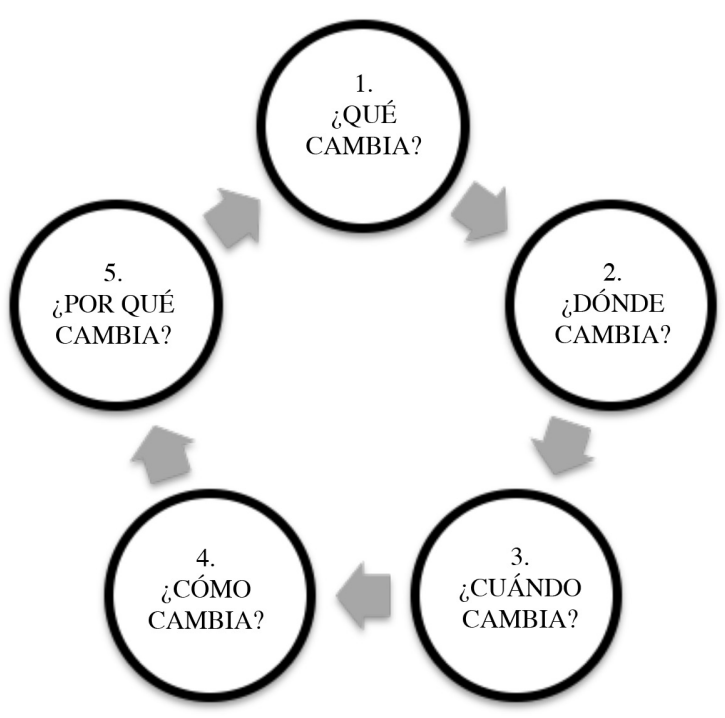

Fuente: Elaboración propia a partir de (Guhl, 2008).

La tipología propuesta permite identificar las fuerzas responsables del cambio en el paisaje. En un paisaje natural, los impulsores del cambio son predominantemente factores biofísicos mientras que en los domesticados y los fabricados prevalecen los impulsores de carácter humano, a partir de sus 
actividades productivas (agrícola, industrial, comercial). La problematización a la que se aproxima el enfoque, es decir, el análisis de la transformación del paisaje, se puede centrar en las siguientes interrogantes que se pretenden responder (Ver Figura No. 1): ¿qué cambia? ¿dónde cambia? ¿cuándo cambia? ¿cómo cambia? ¿por qué cambia? Las respuestas a las tres primeras preguntas permiten documentar y describir el proceso de cambio en el paisaje, mientras que las otras dos respuestas analizan la articulación de los distintos impulsores en el proceso de transformación del uso y de la cobertura del suelo.

De acuerdo con la propuesta LUCC, la cobertura del suelo puede cambiar por dos procesos diferentes: la conversión y la modificación. La conversión se refiere al proceso deliberado de transformar una cobertura en otra, lo que conduce a cambios en los procesos socioeconómicos y ambientales. La modificación se refiere al proceso en el cual se generan ciertos cambios en la cobertura, como resultado de transformaciones en las formas de manejo (Lambim, Rounsevell \& Geist, 2000) (Ver Cuadro No. 2).

Diferencias entre conversión y modificación de la cobertura del suelo

\begin{tabular}{|c|}
\hline CONVERSIÓN DE LA \\
COBERTURA DEL SUELO \\
\hline - La transformación es intencional. \\
- Hay una disrupción de procesos \\
ecológicos. \\
- No se relaciona necesariamente con \\
cambios en el manejo de la tierra. \\
- Hay una transformación directa de la \\
cobertura del suelo. \\
\end{tabular}

\begin{tabular}{|c|}
\hline MODIFICACIÓN DE LA \\
COBERTURA DEL SUELO \\
\hline - Pueden ser cambios involuntarios en \\
la cobertura generados por cambios \\
en el manejo de las mismas. \\
- Los cambios son menos notorios \\
sobre los procesos ecológicos. \\
- Es el resultado de la intervención \\
humana. \\
- Se relaciona con cambios en el \\
manejo de la tierra. \\
- Se presenta una transformación \\
indirecta en la cobertura del suelo. \\
\hline
\end{tabular}

Fuente: Elaboración propia a partir de (Lambim, Rounsevell \& Geist, 2000).

En nuestro criterio, los procesos de conversión y de modificación pueden historiarse y posibilitan el desarrollo de un tipo particular de la historia ambiental, que permite rescatar procesos de antropización vinculados con transformaciones productivas, pero para lograr esto se puede triangular con otros métodos como el uso de mapas históricos y su conversión en mapas georeferenciados.

\section{El enfoque LUCC: las fuerzas y los actores/agentes transformadores del paisaje}

El enfoque LUCC plantea que la mayor parte de los cambios en el uso de la tierra y en la cobertura del suelo son consecuencia de las actividades humanas y que están determinados por el uso de la tierra, es decir, por las presiones productivistas. La evolución en el uso de la tierra debe comprenderse como un proceso complejo, en el que participan fuerzas ambientales, sociales, económicas, políticas e institucionales en escalas diversas. Las distintas fuerzas influyen en las decisiones de los habitantes y de los usuarios de la tierra, quienes finalmente son los transformadores del paisaje (Agarwal, Green, Evans \& Schweik, 2002). 
Figura 2

Fuerzas determinantes en el uso de la tierra y en la cobertura del suelo
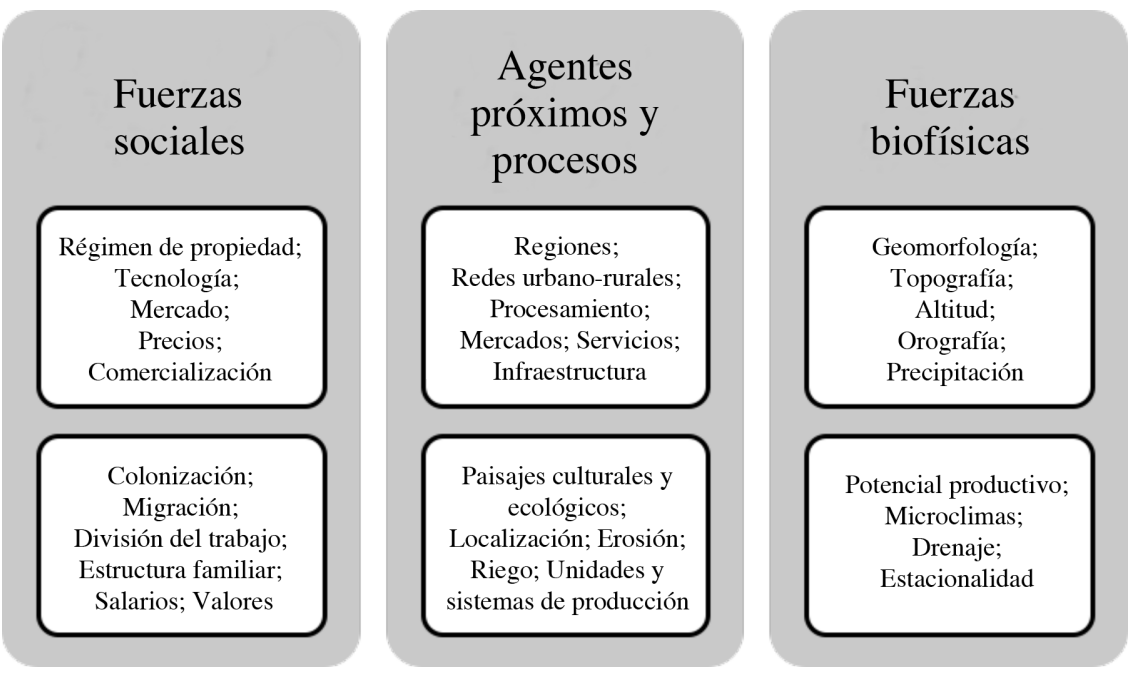

Fuente: Adaptación de Turner, Skole, Sanderson, Günther, Fresco \& Leemans, 1995.

El actor o agente transformador puede ser una persona, una compañía, un gobierno, una entidad o cualquier otro actor involucrado en el manejo de la tierra. A partir del conocimiento de la cobertura del suelo, el usuario puede seleccionar formas de uso de la tierra que alteran la cobertura y que, por lo tanto, pueden alterar también las condiciones económicas, políticas, sociales, institucionales y ambientales. Los actores/agentes se enfrentan constantemente a estos cambios y deben recurrir a la toma de decisiones con respecto al manejo de la tierra y sus recursos (McConnel, 2001). Es importante aclarar que el cambio en la cobertura del suelo puede producirse también por procesos naturales, pero en este caso tiende a ser lento y gradual, aunque también se pueden dar cambios repentinos y rápidos.

No es sencillo clarificar cuál(es) son las fuerzas que determinan las transformaciones del paisaje, debido al principio de la coevolución entre la sociedad y la naturaleza (Ver Figura No. 2). En muy pocas ocasiones solo una provoca el cambio, pues los impulsores generalmente actúan en conjunto (Gueist \& Lambim, 2002). Por eso, las transformaciones varían según el contexto social, económico, político y cultural de las regiones o de los lugares (Ojima, Galvin \& Turner, 1994) y, desde nuestra perspectiva, esto debe proyectarse a una multiescala local, regional, nacional, transfronteriza, internacional, transnacional y global. Lo que sí es claro es que la fuerza institucional es determinante en las transformaciones del paisaje, pues las instituciones tienden a desarrollar regulaciones y normativas para el manejo de los recursos naturales y de los servicios ambientales, que conducen a configuraciones constantes.

\section{Una propuesta de triangulación de enfoque: LUCC y una definición sociohistórica de paisaje}

Es importante triangular la propuesta LUCC con una definición sociohistórica de paisaje, como la que plantea Enric Tello (1999), para quien el paisaje es una construcción humana y un aspecto del territorio. El paisaje existe en la medida que alguien lo mira y lo interpreta para desarrollar algún propósito (económico, estético, lúdico, etc.). El paisaje, según Tello (1999), no existe sin la mediación del 
ojo, la mente y la mano. Como marco de la actividad humana y escenario de su vida social, el paisaje agrario y los paisajes humanos en general son una construcción histórica que resulta de varios factores: la interacción entre los factores bióticos y abióticos del medio natural, los usos de esas capacidades para sustentar el metabolismo económico de las sociedades humanas y los impactos duraderos de esa intervención antrópica sobre el medio. Sin intervención antrópica ni fines humanos no habría entonces paisajes, sino ecosistemas (Tello, 1999).

La coevolución entre sociedad y naturaleza, en el caso particular de los agroecosistemas, deja una huella visible: los paisajes (Guzmán \& González de Molina, 2009) que se pueden reconstruir históricamente, visto el paisaje como la expresión territorial que cualquier sociedad mantiene con los sistemas naturales (Tello, Garrabou \& Cussó, 2007), por lo que algunas de estas relaciones se pueden modelar.

El paisaje es, entonces, una construcción social e histórica ubicada en un tiempo/espacio que, desde una perspectiva (de)constructivista, se caracteriza por la relación existente entre el espacio/ territorio, la territorialidad, una región, un lugar, que no puede dejar de lado los elementos culturales/ subjetivos. En su estudio, hay que tomar en consideración que pueden existir diferentes niveles sociohistóricos de paisajes: puede tratarse de un paisaje socioeconómico, de un paisaje ecológico, de un paisaje institucionalizado o de un paisaje imaginado, pero la conformación de un paisaje solamente es completa cuando se imbrican todos los niveles en una espacialidad relacional entre lo local $\leftrightarrow$ regional $\leftrightarrow$ nacional $\leftrightarrow$ fronterizo $\leftrightarrow$ transnacional $\leftrightarrow$ global. (Cfr. Viales, 2010).

\section{El enfoque LUCC y la modelación}

La mayoría de investigaciones sobre cambio en la cobertura del suelo y uso de la tierra se han basado en la modelación. Los modelos son representaciones simplificadas de la realidad, que se concentran en las relaciones determinantes del fenómeno estudiado, por lo que se encuentran condicionados. Uno de los principales objetivos de la propuesta LUCC es obtener modelos de transformaciones regionales y globales del paisaje, para intentar predecir los cambios y el impacto de los diferentes impulsores sobre la evolución de este. Los modelos sirven, por lo tanto, como un experimento para probar las nociones científicas de procesos fundamentales y para aumentar las comprensiones de las variables críticas y sus relaciones.

De acuerdo con los impulsadores de la propuesta, los modelos deben responder a una de las siguientes preguntas:

1. ¿Cuáles variables ambientales y culturales contribuyen en mayor medida a explicar el cambio en la cobertura del suelo y por qué?

2. ¿Qué lugares se ven afectados por el cambio de cobertura y por qué?

3. ¿A qué tasa progresan los cambios de la cobertura del suelo y cuándo?

Las técnicas para modelar los cambios en la cobertura del suelo y en el uso de la tierra son muy distintas; la programación lineal, la optimización y la modelación estadística son solo algunos ejemplos. Hay modelos de evolución del paisaje que se interesan en las tasas de cambio mientras que otros se concentran en los lugares donde ocurren los cambios. Desde la década de 1990 hasta la actualidad, han existido avances en la modelación del proceso de cambio en el uso de la tierra y cobertura del suelo (Mortimore, Magatte, Mahamane, Rostom, Serra \& Turner, 2005). Sin embargo, todavía persisten las limitaciones que tienen que ver más con la comprensión del proceso que con la carencia de tecnologías y métodos. Para llenar ese vacío interpretativo, la historia ambiental puede constituir un insumo importante. Una de las limitantes es que no siempre se incorpora en los modelos el proceso de toma de decisiones de los actores/agentes que utilizan la tierra y transforman el paisaje; y otra es que las unidades de modelación no siempre corresponden a la decisión de uso de la tierra por parte de los usuarios. 


\section{El enfoque LUCC y la actividad agrícola}

El enfoque LUCC ha trabajado las transformaciones del paisaje básicamente a partir del urbanismo y de las actividades agrícolas. En lo que respecta a la actividad agrícola, su acercamiento ha sido a partir de dos variables: la intensificación y la comercialización. Los usuarios de la tierra, como ya se mencionó, se enfrentan a diario con cambios y, para responder a estos, desarrollan estrategias; tales como nuevas prácticas culturales o la introducción de tecnología. La manera en que se da el cambio agrícola es diversa, como también diversa es la transformación del paisaje asociada a esta. El cambio en la agricultura y los cambios en la cobertura resultan de dos tipos principales de transformación: la tecnológica y la estructural. En la primera, el cambio resulta de la transformación de tecnologías y de estrategias de manejo de la tierra; y en la segunda, las transformaciones resultan de los cambios en las condiciones sociales y económicas.

La intensificación en la agricultura obliga a aumentar la productividad de la tierra y casi siempre está vinculada con el aumento de la participación de la producción agrícola en el mercado (Ver Cuadro No. 3). Se pasa, paulatina o abruptamente, de una economía de subsistencia a una economía comercial, de modo que la relación agricultura/evolución del paisaje no se puede comprender al margen del mercado (interno-externo).

Cuadro 3

Factores de la intensificación agrícola
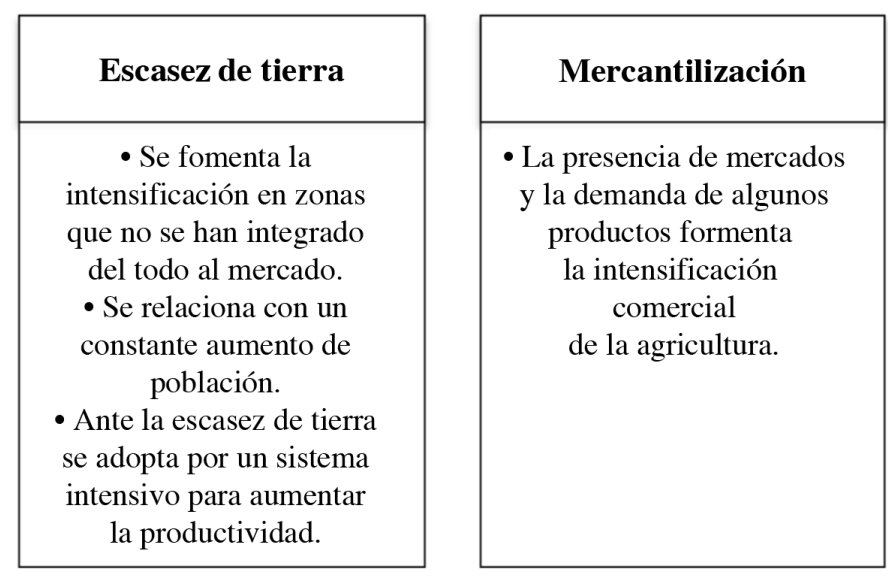

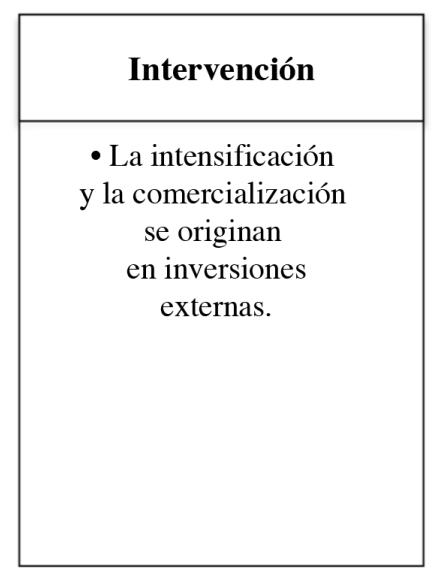

Fuente: Elaboración propia a partir de (Guhl, 2008).

La intensificación y la comercialización llevan entonces a un cambio agrícola que solo se puede estudiar desde una perspectiva integral que combine elementos socioeconómicos, políticos, técnicos, institucionales, ambientales y de mercado. Un sistema agrícola es, por consiguiente, el resultado de variables materiales, variables estructurales y de comportamiento individual y comunitario, por lo que cada uno tiene sus propias particularidades. Lo que se traduce en un proceso complejo que conduce a transformaciones paisajísticas de gran envergadura, que están relacionadas con las decisiones de los que tienen acceso a la tierra y, como se señaló antes, los que no tienen acceso a la tierra, pero son los que promueven muchas de las decisiones. 


\section{El enfoque LUCC y sus limitantes: la escala y las fuentes}

Las transformaciones del paisaje, según el enfoque LUCC, no se pueden comprender al margen de las relaciones espaciales y temporales entre el uso de la tierra y la cobertura del suelo. El cambio en el uso de la tierra y la cobertura del suelo es necesariamente un proceso multiescalar, pues muchas actividades humanas y procesos ecológicos tienen causas y consecuencias en diferentes escalas. A su vez, cada escala se particulariza por unidades y variables propias, lo que obliga a analizar sus interacciones (Gibson \& Ahh, 2000). Lo multiescalar complejiza entonces la investigación, pues se tiene que integrar sistemas sociales y naturales que casi siempre operan en escalas espaciales y temporales diferentes, por lo que la interrelación, en principio, se desconoce. Los impulsores del enfoque LUCC reconocen que lo anterior es una limitante importante, pues es difícil identificar las escalas espaciales y temporales correctas para un proyecto determinado (Ojima, Galvin \& Turner, 1994).

Otra limitante es la propia reconstrucción de los procesos. Para que el análisis sea exitoso, se requiere buscar, compilar y analizar, mapas de cobertura de diferentes momentos a lo largo del tiempo. Las fotografías aéreas y los mapas satelitales se han convertido en herramientas importantes. No obstante, en la mayoría de los casos son muy contemporáneos, por lo que habría que valerse de otros métodos para analizar el cambio en la cobertura del suelo y el uso de la tierra. La Historia -como disciplina- podría contribuir en la reconstrucción, con la consulta de fuentes primarias; aunque es posible que en la mayoría de casos solo se obtengan aproximaciones para el cambio en la cobertura del suelo y el uso de la tierra. De esta forma, la fotografía se constituye en una fuente histórica de primer orden.

Como se mencionó anteriormente, al enfoque LUCC le interesa estudiar los impulsores que determinan el cambio de la cobertura del suelo -analizando los procesos de toma de decisiones de los usuarios de la tierra- para identificar los factores que determinaron en las decisiones. Es posible que algunos documentos históricos permitan acercarse a dicha identificación, desde la mediana o la larga duración. Desde la geografía histórica se han realizado también relevantes avances en la reconstrucción de paisajes. El trabajo en equipo de historiadores y geógrafos ha permitido mapear algunos cambios en paisajes regionales que hasta hace poco tiempo se hubiera considerado imposible. Sin duda, el éxito depende en buena medida de las fuentes y de su tratamiento a partir de métodos novedosos, como la cartografía histórica geo-referenciada, como han planteado Juan José Marín y Hubert Vargas (2011).

\section{Conclusión}

El enfoque LUCC podría ser útil para la historia ambiental y aún más si se triangula con otras propuestas, pues, como ya se apuntó, presenta algunas limitaciones. Resulta necesario tratar de reconstruir paisajes, pero sin estancarse en la mera descripción sino con interpretación y análisis. Como se apuntó anteriormente, medir los cambios en la cobertura del suelo y el uso de la tierra no es un trabajo sencillo, aunque desde la interdisciplinariedad, los resultados pueden ser valiosos. Esta joven propuesta cuenta cada vez con más seguidores críticos que promueven nuevas alternativas, sobre todo en los procesos de modelación.

El enfoque LUCC es una iniciativa que nace en los Estados Unidos y se ha ido extendiendo a otros países europeos y asiáticos. Es posible que con el transcurso del tiempo aparezcan proyectos en otras latitudes que decidan adaptar esta propuesta, para estudiar las transformaciones del paisaje. Desde nuestro punto de vista, en América Latina se puede adaptar/adoptar este enfoque para el estudio de los procesos de agriculturización (Cfr. Montero \& Viales, 2014) y de industrialización, a partir de la coevolución entre sociedad y naturaleza.

Las fuentes históricas en los archivos nacionales y en otros centros documentales nos pueden ayudar a reconstruir elementos físicos y humanos de los paisajes en el pasado que nos 
permitan comprender la dinámica de los paisajes actuales y los del futuro. El historiador como científico social puede ser un actor clave en la propuesta LUCC, en la medida en que con las herramientas y técnicas de la historia logre historiar el paisaje, un paisaje que claramente tiene componentes físicos -que comprenderán muy bien los geólogos, los físicos, los biólogos, los agrónomos- pero uno también con claros componentes sociales, que forman parte del territorio académico de los historiadores.

\section{Referencias}

Agarwal Chetan; Green, Glen; Morgan, Grove; Evans, Tom y Schweik, Charles (2002). A Review and Assessment of Land-Use Change Models: Dynamics of Space, Time, and Human Choice. USDA. USA. 62 p.

Gibson, Clark; Ostrom, Elinor y Ahh, T.K. (2000). The concept of scale and the human dimensions of global change: a survey. Ecological Economics, (32), 217-239.

Gueist, Helmut y Lambim, Eric (2002). Proximate Causes and Underlying Driving Forces of Tropical Deforestation. BioScience, 52 (2),143-150.

Guhl, Andrés (2009). Café y cambio de paisaje en Colombia, 1970-2005. Medellín, Universidad EAFIT.

Guzmán, Gloria y González de Molina, Manuel. (2009). Preindustrial agriculture versus organic agriculture. The land cost of sustainability. Land Use Policy, 26 (2), 502-510.

Lambim, E.F; Rounsevell, D.A. y Geist, H.J. (2000). Are agricultural land use models able to predict changes in land-use intensity? Agriculture, Ecosystems and Environment, 81 (1-3), 231-331.

Marín, Juan José y Vargas, Hubert (2011). La cartografía histórica georeferenciada. Una visión desde Centroamérica para analizar los espacios y sus trayectorias. Propuesta de trabajo. En Viales, Ronny; Díaz David y Franzé, Javier (Comps), América Latina: conceptos y conflictos (pp. 45-86), San José: Editorial Nuevas Perspectivas.

McConnell, William (2001). Agent-Based Models of Land-Use and Land-Cover Change. Report and Review of an International. LUCC Report Series, (6), 1-129.

Montero, Andrea y Viales, Ronny (2014). Agriculturización' y cambios en el paisaje. El banano en el Atlántico/Caribe de Costa Rica (1870-1930). Revista de Historia Ambiental Latinoamericana y Caribeña (HALAC), III (2), 310-338.

Mortimore, Michael; Ba, Magatte; Mahamane, Ali; Rostom, R.S., Serra del Pozo, Pau y Turner, Beryl (2005). Changing systems and changing landscapes: Measuring and interpreting land use transformation in African drylands. Geografisk Tidsskrift-Danish Journal of Geography,105 (1), 101-118.

Ojima D.S; Galvin, K.A. y Turner, B.L. (1994). The Global Impact of Land Use Change. BioScience, 44 (5), 300-304.

Tello, Enric (1999). La formación histórica de los paisajes agrarios mediterráneos: una aproximación coevolutiva. Historia Agraria, (19), 195-212.

Tello, Enric; Garrabou, Ramón y Cussó Xavier (2003) Tras las huellas ecológicas del Metabolismo Social. Una propuesta metodológica para analizar el paisaje como humanización del territorio. III Seminario sobre evolución del territorio como punto de encuentro transdisciplinar, organizado por la Universidad de las Islas Baleares. Palma de Mallorca, (20), 1-25.

Turner, B.L (1995). Land Use and Land Cover Change. Science-Research Plan. IGBP/HDP. USA. 131 p.

Tuner, B.L; Meyer, B. y Skole, D. (1994). Global Land-Use/Land Cover. Towards an Integrated Study. Ambio, 23 (1), 91-95.

Viales, Ronny (2010). La región como construcción social, espacial, política, histórica y subjetiva. Hacia un modelo conceptual/relacional de historia regional en América Latina. Geopolítica(s), $1(1), 157-172$. 
